\title{
Numerical determination of plastic collapse loads for sections under concentrated transverse forces
}

\author{
Gabriel B. dos Santos*,a Leroy Gardner ${ }^{\mathrm{a}}$, Merih Kucukler ${ }^{\mathrm{a}}$ \\ amperial College London, Dept. of Civil and Environmental Engineering, United Kingdom \\ g.santos14@imperial.ac.uk, leroy.gardner@imperial.ac.uk,merih.kucukler10@imperial.ac.uk
}

\begin{abstract}
Structural steel design codes generally use design methods requiring the determination of two key reference loads: (i) the plastic collapse load and (ii) the elastic buckling load. Using these two key reference loads, the element slenderness can be determined and member resistances can be obtained through buckling curves. Both of these reference loads can be estimated either through simplified analytical expressions or numerical analysis. In the cases of uniform loading, regular geometry and straightforward boundary conditions, the former approach is well suited, while for problems leading to complex non-uniform stress distributions, such as members under concentrated transverse loading, numerical solutions are often necessary.

Using numerical methods, the plastic collapse loads can be obtained through Materially Nonlinear Analyses (MNA). However, since the development of a plastic collapse mechanism brings about large localised strains and extensive plasticity, a MNA can abort prematurely due to its failure to satisfy the necessary convergence criteria or the obtained load-deformation path can flatten out only after unrealistically large deformations, resulting in misleading predictions of the plastic collapse loads. In this paper, an extrapolation technique is recommended for the determination of plastic collapse loads from MNA for plate elements and cold-formed sections under concentrated transverse loading. The proposed procedure is based on the Modified Southwell (MS) Plot, originally proposed for the identification of critical loads.
\end{abstract}

Keywords: concentrated transverse loading, Modified Southwell Plot, plastic collapse load, web crippling

\section{INTRODUCTION}

Structural steel design standards generally use design methods utilising two key reference loads: (i) the plastic collapse load and (ii) the elastic buckling load, from which the member slenderness and hence the member resistance can be determined. Both of these reference loads can be obtained either through simplified analytical expressions or numerical analysis. For structural elements under uniform loading and with regular geometry and boundary conditions, the former is suitable, while for those with complex non-uniform stress distributions, which may arise due to concentrated transverse loading, numerical analyses are often necessary.

For the prediction of the plastic collapse loads of elements under concentrated loading, Materially Nonlinear Analyses (MNA) with an elastic perfectly-plastic material model have typically been adopted in the literature $[1,2]$. However, in many cases, the determination of the actual plastic collapse load from a MNA is not straightforward since (i) a MNA can abort prematurely because of its failure to satisfy the necessary convergence criteria or (ii) the obtained load-deformation path can flatten out only after unrealistically large deformations have been reached, with the result that the corresponding load does not reflect the nature of collapse mechanism that would arise in practice. In this paper, different methods for the consistent determination of the realistic plastic collapse loads from MNA are investigated. Initially, finite element models of plates and coldformed steel elements are developed and their validation against the results of an existing experimental study is presented. Potential problems in the determination of plastic collapse loads from load-deformations graphs are described and different extrapolation techniques utilised to predict the plastic collapse loads are analysed. Finally, a practical approach based on the Modified 
Southwell (MS) Plot is recommended for the determination of the plastic collapse loads from the MNA of structural steel elements.

\section{DESCRIPTION AND VALIDATION OF FINITE ELEMENT MODELS}

In this section, the finite element modelling approach adopted in this study is described and its validation against the results of an experimental study from the literature is presented. In the subsequent sections, MNA using the finite element models of plates and cold-formed sections are performed for the prediction of their plastic collapse loads.

\subsection{Numerical model}

Finite element models of steel plates and cold-formed steel beams were created through the finite element software Abaqus [3]. The general-purpose shell element S4R, which is a four-noded doubly-curved shell element with reduced integration, was used to model the investigated steel plates and cold-formed steel beams. The accuracy of this element type has been illustrated in previous investigations of plates under compressive edge loading and cold-formed beams under transverse loads $[2,4]$. An elastic-perfectly plastic material model was utilised in the finite element models, where the Young's modulus was taken as $200 \mathrm{GPa}$ and the Poisson's ratio was assumed to be equal to 0.3 . In the case of steel plates, simply-supported boundary conditions were modelled on the longitudinal edges where the loads are applied, restraining the out-of-plane displacements $(U 1)$ along the whole length and restraining the longitudinal displacements (U3) only at the mid-length nodes, as illustrated in Fig. 1. The loading conditions of the plate are shown in Fig. 1, where $L$ is the length of the plate, $s_{s}$ is the loaded length and $\delta_{\mathrm{h}}$ is the maximum value of the imperfection. A mesh with an element size equal to $5 \mathrm{~mm}$ was adopted for all the investigated steel plates, following a mesh sensitivity study.

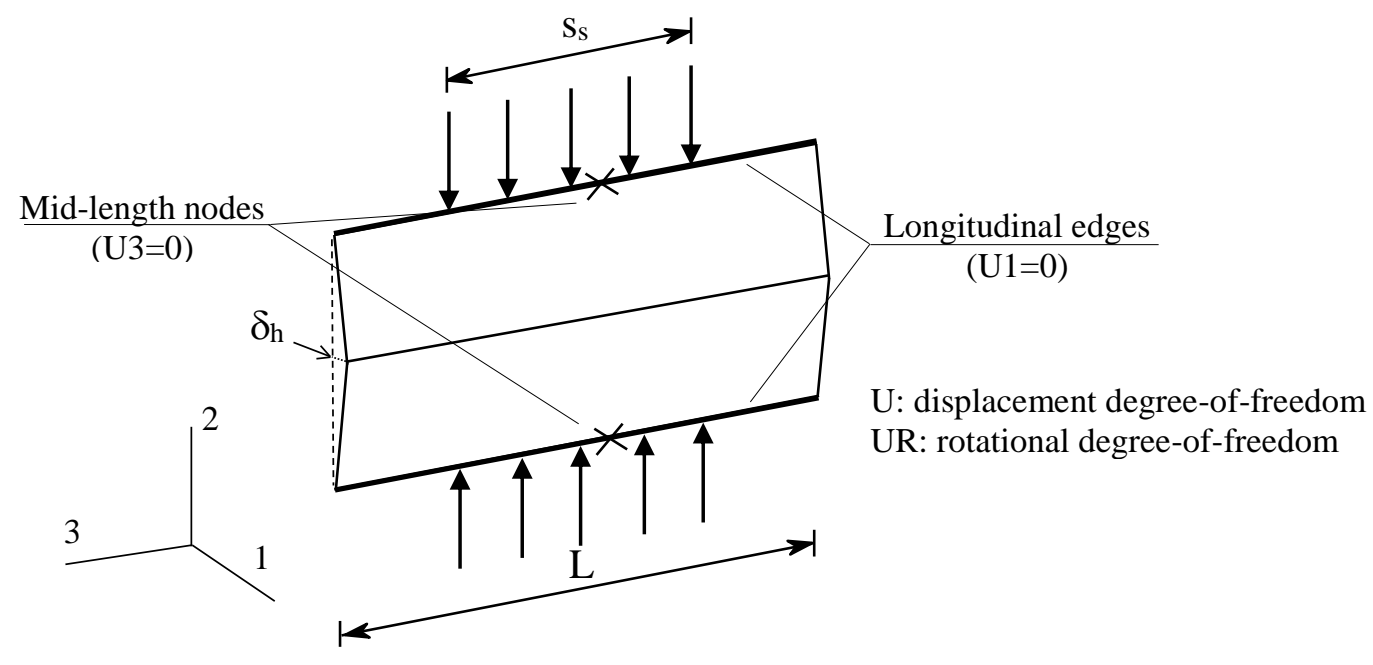

Fig. 1. Loading and boundary conditions adopted in the finite element model of a plate under in-plane loading.

Finite element models of cold-formed steel beams were created replicating the experimental set-up reported by Young and Hancock [5], featuring two back-to-back cold-formed channel sections, a bearing plate under the applied concentrated load at the mid-span to the beams, and support plates at the ends of the beams. The developed finite element model is shown in Fig. 2. An eight-node linear hexahedral solid element C3D8 was employed to model both the bearing and support plates. The element size was taken as equal to $4.5 \mathrm{~mm}$ in the flat regions of the cold-formed beams and refined to the $1 \mathrm{~mm}$ around the corners. Surface-to-surface contact with a friction coefficient of 0.4 and a contact stiffness of $4000 \mathrm{~N} / \mathrm{mm}$ was employed between the bottom face of the bearing block and the top flanges and corners of the cold-formed beams, enabling a realistic representation of the load transfer into the beams. 

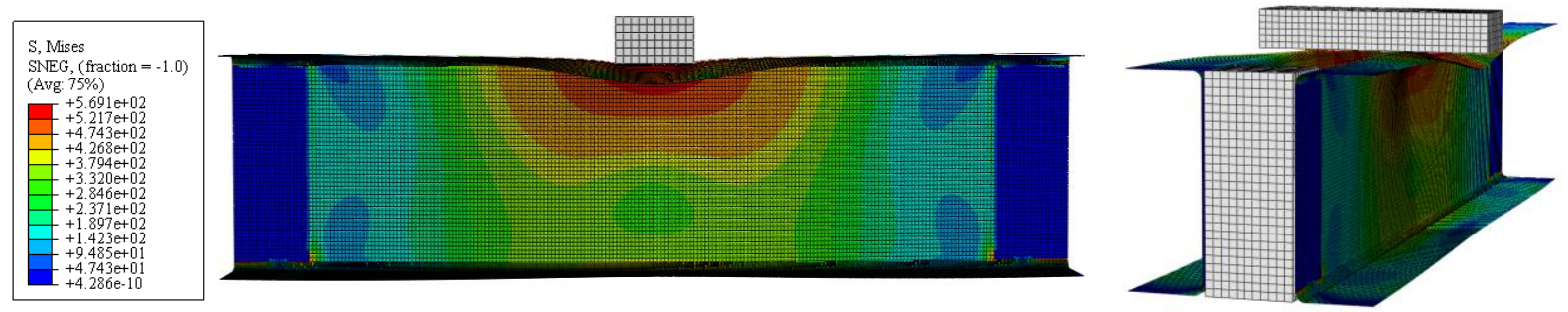

Fig. 2. GMNA simulation of IOF250N90 specimen at failure - von Mises stress distribution.

\subsection{Validation of the adopted finite element modelling approach}

Validation of the finite element model for specimen IOF250N90 tested by Young and Hancock [5] is illustrated in Fig. 3, where the reaction force at the support versus the vertical displacement at the load application point curves obtained through the experiment and numerical model are provided. Note that in the finite element model, the yield stress was taken as equal to $469 \mathrm{MPa}$, which is the $0.2 \%$ proof stress of the cold-formed section obtained from the coupon tests by [5]. As can be seen from Fig. 3, very good correlation between the load-deflection path observed in the test and that obtained through the Geometrically and Materially Nonlinear Analysis (GMNA) of the finite element model of the specimen was obtained, indicating that the adopted finite element modelling approach is able to provide accurate predictions of the actual response of steel elements subjected to transverse loading and that Materially Nonlinear Analysis (MNA) performed through it should provide accurate predictions of plastic collapse loads. The deformed shape of the finite element model of the specimen with the von Mises stress distribution is illustrated in Fig. 2; the deformation pattern matches that observed in the experiment [5].

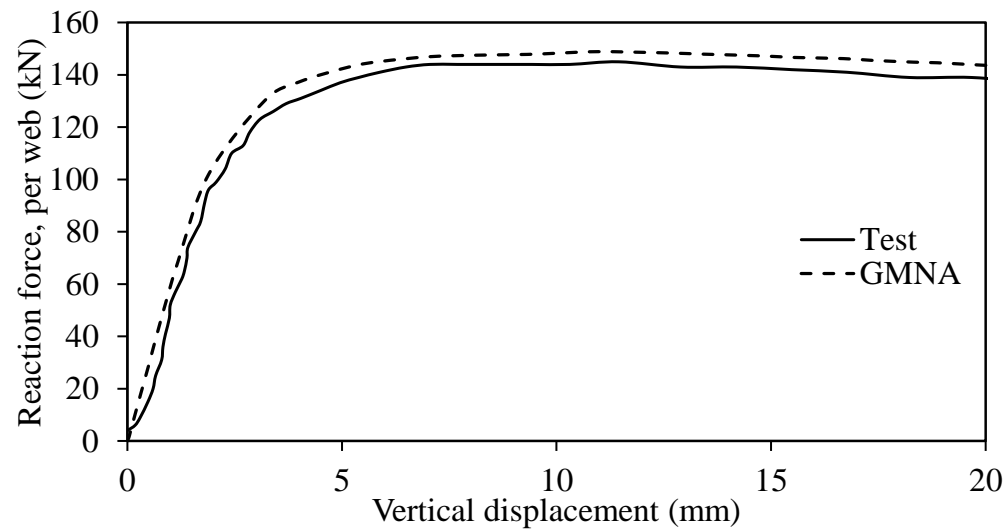

Fig. 3. Load-vertical displacement curves of IOF250N90 specimen [5]: Test vs. GMNA.

\section{POTENTIAL PROBLEMS WITH THE DETERMINATION OF PLASTIC COLLAPSE LOADS FROM MATERIALLY NONLINEAR ANALYSIS (MNA)}

As previously mentioned, in many cases, the determination of plastic collapse loads of structural steel elements from MNA can be challenging since the MNA (i) may provide a load-deformation curve flattening out only after unrealistically large deformations or (ii) may be terminated prematurely due to its failure to satisfy the necessary convergence criteria. In this section, these two main problems are described. Alternative approaches that can be employed to determine the plastic collapse loads from MNA even in the cases of the occurrence of these problems will be discussed in the following sections, developing a robust approach able to estimate plastic collapse loads of steel elements using load-deformation curves obtained from MNA.

\subsection{Mechanism associated with unrealistic deformations}

In this section, one of the two primary problems encountered in the determination of plastic collapse loads from MNA, which is associated with the development of collapse mechanisms only after unrealistically large deformations, is addressed by analysing a rectangular steel plate subjected to compressive loading on the longitudinal edges with different loading lengths $s_{s}$. The studied plate 
has a yield stress $f_{y}$ equal to $400 \mathrm{MPa}$, a length $\mathrm{L}$ of $900 \mathrm{~mm}$, a height of $300 \mathrm{~mm}$, a thickness t of 4 $\mathrm{mm}$ and an initial imperfection $\delta_{\mathrm{h}}$ of $0.01 \mathrm{~mm}$, as shown in Fig. 1.

The load-mid-span vertical displacement curves obtained through the MNA are illustrated in Fig. 4 (a) for various loaded lengths $s_{s}$. As can be seen from the figure, for loaded lengths $s_{s}$ less than 880 $\mathrm{mm}$, the applied loading continues to increase even after unrealistically large deformations, for which collapse mechanisms with the yielding of excessive regions were observed in the finite element models. Since these types of mechanisms are not representative of the actual collapse mechanisms observed in physical experiments [5], simply extracting the ultimate loads from the MNA as the plastic collapse loads would be incorrect.

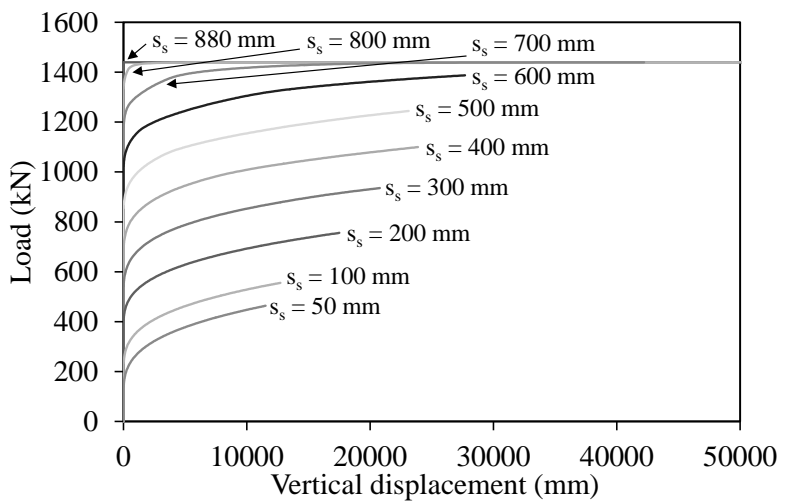

(a)

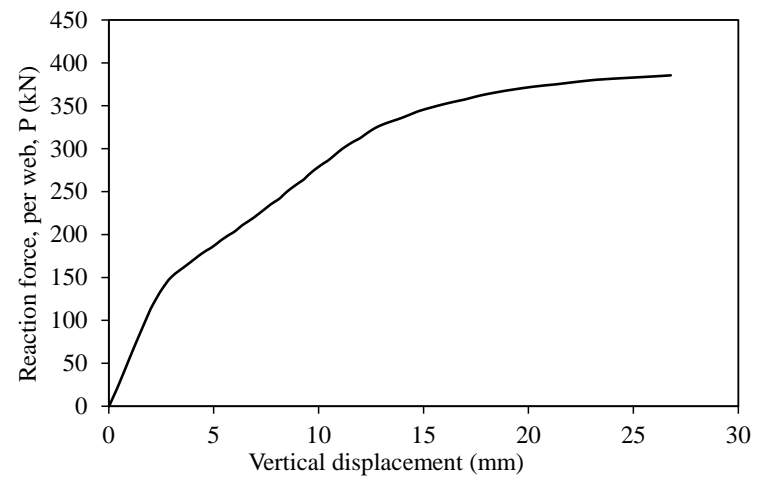

(b)

Fig. 4. Load-displacement curves from MNA of (a) plates with initial imperfections of $0.01 \mathrm{~mm}$ under partial bearing loads and (b) IOF250N90 cold-formed beam specimen [5].

\subsection{Premature termination of the analysis}

Another common problem encountered in the determination of plastic collapse loads from MNA is the premature termination of the analysis, which is illustrated in this section by performing an MNA of the cold-formed steel beam specimen IOF250N90 of [5] previously used in the validation of the finite element modelling approach adopted in this study. The load-displacement path obtained through the MNA of the cold-formed beam is shown in Fig. 4 (b), where it may be seen that the analysis stopped before the load-displacement curve flattens out due to its failure to satisfy the necessary convergence criteria prior to the formation of the collapse mechanism. Convergence problems, which can result for a number of reasons such as inadequate values for solver parameters, extreme local deformations and local instabilities, are also commonly encountered in MNA of shell structures, for which different extrapolation techniques were developed for the prediction of plastic collapse loads [6].

\section{EXTRAPOLATION TECHNIQUES FOR THE ESTIMATION OF PLASTIC COLLAPSE LOADS}

In this section different techniques that can be used for the determination of plastic collapse loads from the MNA of steel elements are presented.

\subsection{Southwell Plot}

Southwell [7] observed that for small displacements, the load-lateral deflection curve of an imperfect column can be approximated by a hyperbolic function having an upper bound value asymptotic to the well-known Euler critical load, as shown in Fig. 5 (a). To obtain the Euler critical load, Southwell proposed to plot the expression obtained by the division of the lateral deflection $\delta$ by the applied load $P$ (i.e. $\delta / P$ ) versus the lateral deflection $\delta$. Within the small displacement region, this relationship can be approximated by a straight line with a slope equal to the inverse of the critical load (i.e. $1 / P_{c r}$ ), which intersects the deflection axis at a value equal to the initial imperfection $w_{0}$, as shown in Fig. 5 (b). This approach, originally used for the determination of $P_{\mathrm{cr}}$, will be employed in following section to obtain the plastic collapse loads from MNA. 

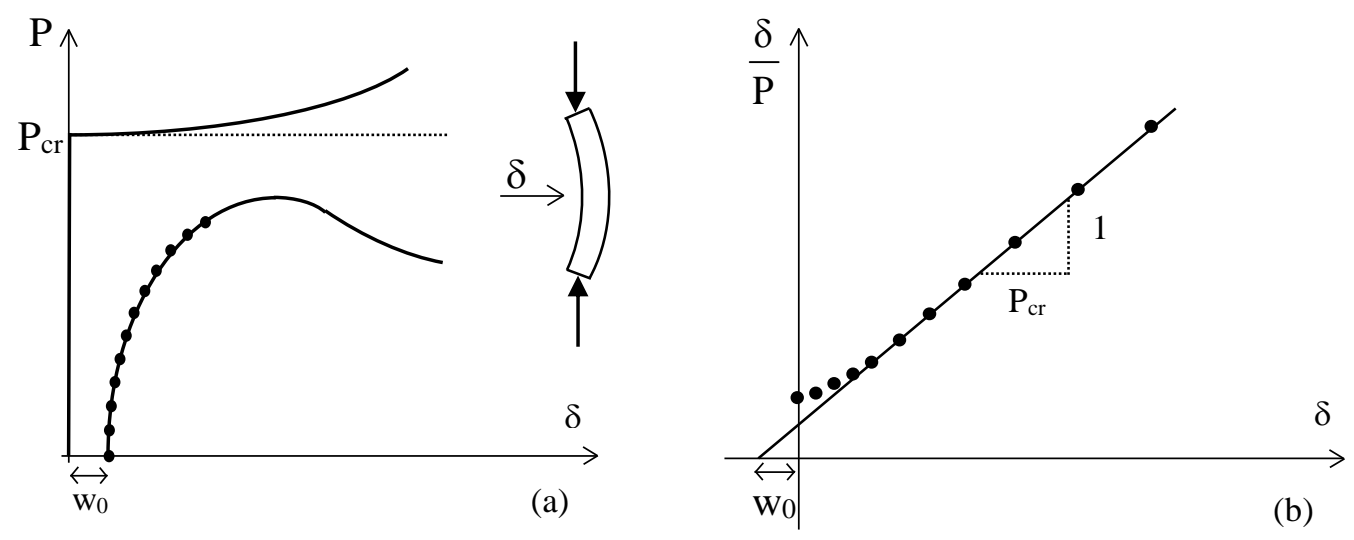

Fig. 5. (a) Load-displacement curve of a column adapted from [7]; (b) Southwell plot adapted from [8]

\subsection{Modified Southwell Plot (MS plot)}

For the determination of the Euler critical load, Horne and Merchant [8] recommended an alternative approach shown in Fig. 6 (a), which is referred to as the Modified Southwell (MS) Plot in this paper. In the MS Plot, (i) the axial load $P$ is plotted against the ratio $P / \delta$, (ii) a series of regression lines are determined using three consecutive values on the $P-P / \delta$ curve and the points where these regression lines intersect the vertical axis are taken as the critical load estimates $P_{\mathrm{MS}, \mathrm{i}}$ and (iii) the lowest value $P_{\mathrm{MS}}$ is taken as the Euler critical load $P_{\text {cr. }}$ A very similar procedure to that outlined above and also used by Holst et al. [9] will be adopted in the following section of this paper for the determination of plastic collapse loads from MNA.

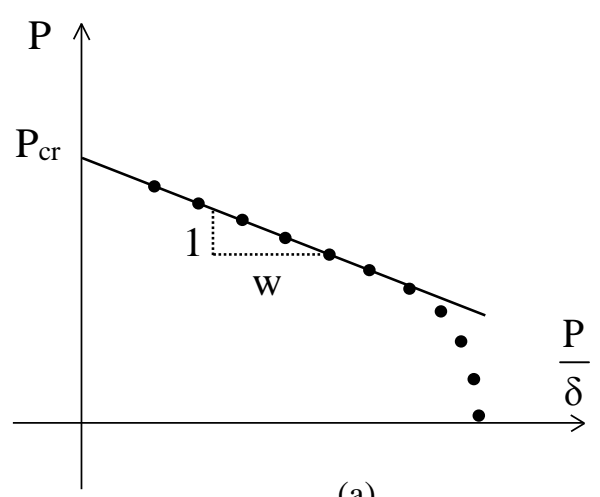

(a)

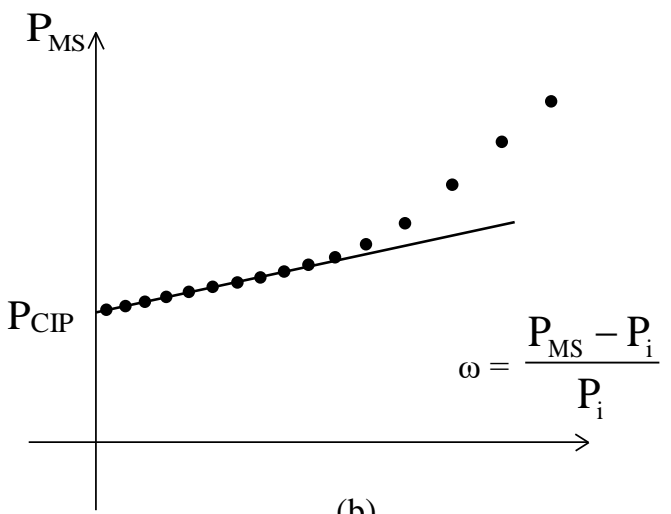

(b)

Fig. 6. Modified Southwell Plot [8] and Convergence Indicator Plot [9].

\subsection{Convergence Indicator Plot (CIP)}

To determine a plastic limit load from MNA, the Convergence Indicator Plot (CIP) was proposed by Doerich and Rotter [6] as an alternative to the MS Plot. As shown in Fig. 6 (b), in the CIP, the vertical axis represents the plastic limit load predicted by the regression lines of the MS plot $P_{\mathrm{MS}, \mathrm{i}}$, while the horizontal axis features the parameter $\omega$ which is equal to the difference between the plastic limit load of each regression line of the MS Plot $P_{\mathrm{MS}, \mathrm{i}}$ and the total applied load at the end of each increment of the MNA $P_{\mathrm{i}}$, normalised $P_{\mathrm{i}}$ (i.e. $\left.\omega=\left(P_{\mathrm{MS}}-P_{\mathrm{i}}\right) / P_{\mathrm{i}}\right)$. When the predicted plastic load from the MS plot $P_{\mathrm{MS}, \mathrm{i}}$ approaches the total applied load $P_{\mathrm{i}}, \omega$ approaches zero and the plastic collapse load $P_{\mathrm{CIP}}$ is estimated through linear extrapolation. The parameter $\omega$, therefore, provides an indication with respect to the proximity of the total applied load at the end of each increment to the plastic collapse load determined through the MS Plot. Holst et al. [9] used the CIP for the prediction the plastic loads of steel elements, showing that the actual collapse loads can be accurately predicted provided $\omega$ values of less than about 3 and 0.5 for plates and shells respectively can be achieved in the analyses.

\section{APPLICATION TO ELEMENTS UNDER CONCENTRATED LOADING}

In this section, the advantages and limitations of the extrapolation techniques described in the previous section when applied to the determination of plastic collapse loads for elements under 
concentrated loading from MNA are investigated. A rectangular simply-supported plate subjected to compressive edge loading with a loading length $s_{\mathrm{s}}$ of $400 \mathrm{~mm}$ is analysed. The considered plate has a yield stress $f_{y}$ equal to $400 \mathrm{MPa}$, a length $\mathrm{L}$ of $900 \mathrm{~mm}$, a height of $300 \mathrm{~mm}$, a thickness t of $4 \mathrm{~mm}$ and an initial imperfection $\delta$ of $0.01 \mathrm{~mm}$.

\subsection{Southwell Plot}

Fig. 7 shows the Southwell Plot obtained from the MNA of the considered plate. In Fig. 7 (a), the vertical displacement at the middle of the loaded length is limited to $6 \mathrm{~mm}$, while in Fig. 7 (b), the displacements up to $100 \mathrm{~mm}$ are considered. In both figures, the regression lines are determined in accordance with the Southwell approach described in the previous section. Depending on the considered displacement magnitude, different values of plastic collapse loads taken as the inverse of the slope of the regression line, are obtained, which are shown in Table 1. In this situation, the Southwell plot does not offer a consistent means of determining the plastic collapse load from an MNA since the result is dependent of the data range considered.

Table 1. Plastic collapse load of a plate under partial bearing load predicted by Southwell plot.

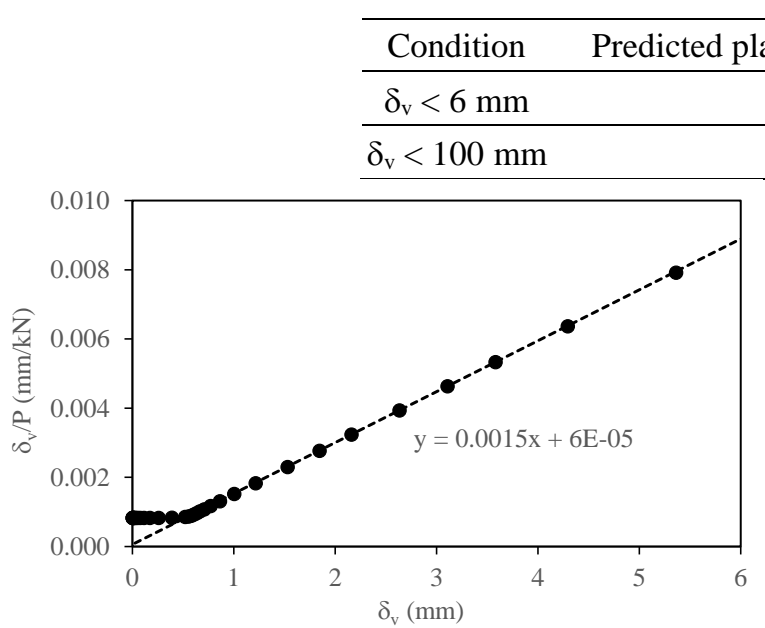

(a)

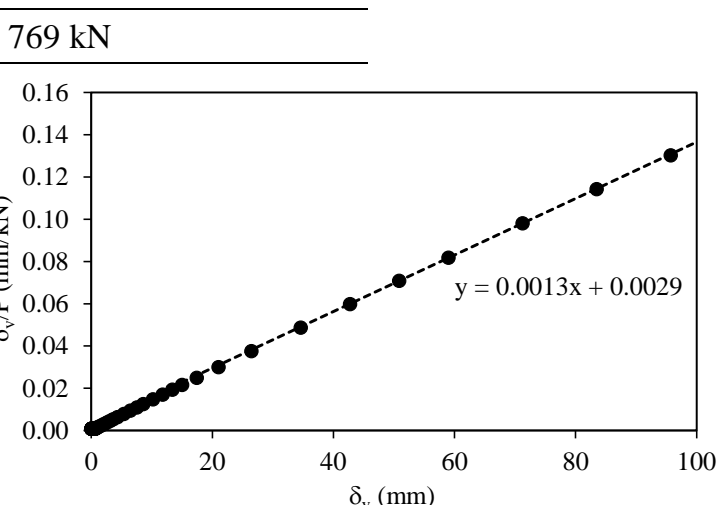

(b)

Fig. 7. Southwell Plot applied to a plate under a partial bearing load of $400 \mathrm{~mm}$ for (a) small displacements and (b) large displacements.

\subsection{Modified Southwell Plot (MS plot)}

The MS Plot of the considered plate, which is shown in Fig. 8 (b), was obtained using the loaddisplacement curve determined through MNA, which is illustrated in Fig. 8 (a). The $P-P / \delta$ curve of the MS Plot has an initial vertical path with a constant $P / \delta_{\mathrm{v}}$ ratio, where the applied load $P$ increases at the same rate as $\delta_{\mathrm{v}}$. A sharp change in the initial path occurs with the spread of yielding and a very clear plateau is observed in the MS Plot, where the displacement $\delta_{\mathrm{v}}$ increases considerably while the applied load $P$ stays almost constant, signifying the development of a mechanism. As shown in Fig. 8 (b), for very small $P / \delta_{\mathrm{v}}$ ratios and large displacements $\delta_{\mathrm{v}}$, a third path is also present in the MS plot where the load $P$ and displacement $\delta_{\mathrm{v}}$ both increase before the termination of the MNA. This third path is associated with the unrealistic spreading of the plastic mechanism at excessively high deformations and should be disregarded; the plastic collapse load should be taken as a load corresponding to the data points within the plateau of the MS plot.

To determine the plastic collapse load $P_{\mathrm{p}}$ from the MS plot, the following procedure is adopted: (i) initially, a series of linear regression lines are obtained through regression analysis using each set of three consecutive data points from the onset of the applied loading, (ii) the plastic load provided by each regression line $P_{\mathrm{MS}, \mathrm{i}}$ is taken as the positive constant of the linear function representing the regression line (i.e. the value where the regression line intercepts the vertical axis), and (iii) finally, if the plastic collapse load provided by the regression line $P_{\mathrm{MS}, \mathrm{i}}$ is smaller than that of the subsequent regression line $P_{\mathrm{MS}, \mathrm{i}+1}, \mathrm{P}_{\mathrm{MS}, \mathrm{i}}$ is assumed as the plastic collapse load provided by the MS plot (i.e. $P_{\mathrm{p}}=P_{\mathrm{MS}, \mathrm{i}}$ if $P_{\mathrm{MS}, \mathrm{i}}<P_{\mathrm{MS}, \mathrm{i}+1}$ ). In cases of $\mathrm{MS}$ plots with more than one plateau, the predicted 
plastic collapse load from the final plateau should be considered. Following the described procedure, the plastic collapse load of $671.6 \mathrm{kN}$ was obtained through the MS plot for the investigated plate, whose corresponding regression line is illustrated in Fig. 8(b).

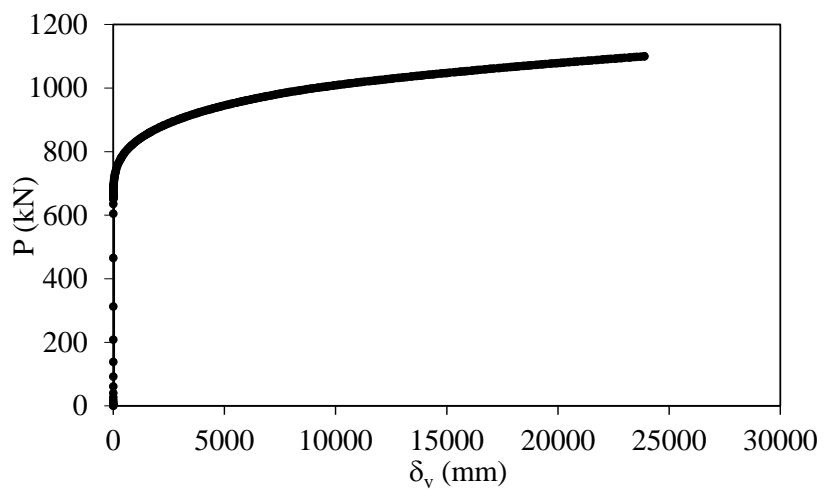

(a) Load-displacement curve

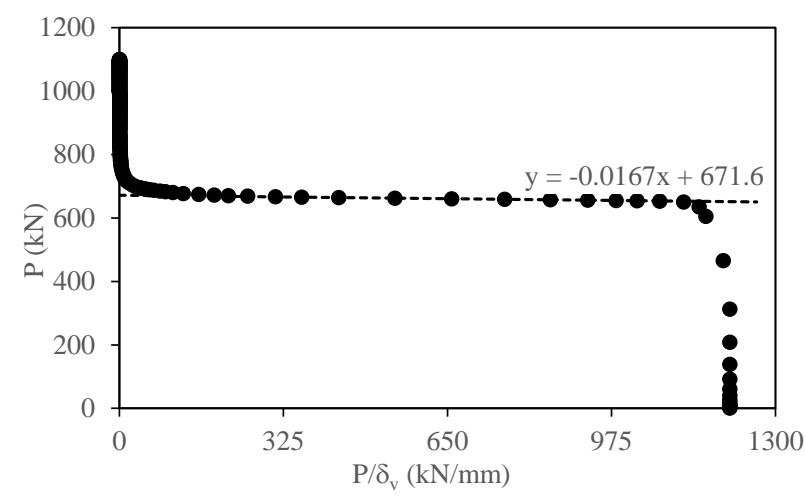

(b) Modified Southwell Plot

Fig. 8. Load-displacement curve and MS Plot for a plate under a partial bearing load of $400 \mathrm{~mm}$.

\subsection{Convergence Indicator Plot (CIP)}

The obtained Convergence Indicator Plot (CIP) for the investigated plate is shown in Fig. 9. In the CIP, the plastic loads are determined from the subsequent regression lines. Each regression line of the MS Plot $P_{\mathrm{MS}, \mathrm{i}}$ is plotted on the vertical axis, while the corresponding values of $\omega_{\mathrm{i}}=\left(P_{\mathrm{MS}, \mathrm{i}}-P_{\mathrm{i}}\right) /$ $P_{\mathrm{i}}$ for each increment (see Fig. 6 (b)) are plotted on the horizontal axis. The plastic collapse load obtained from the CIP $P_{\text {CIP }}$ is taken as the constant term of the function representing the regression line fitted to the three lowest $\omega$ values of the CIP. Only the data points within a range of $0 \leq \omega \leq 3$, were considered.

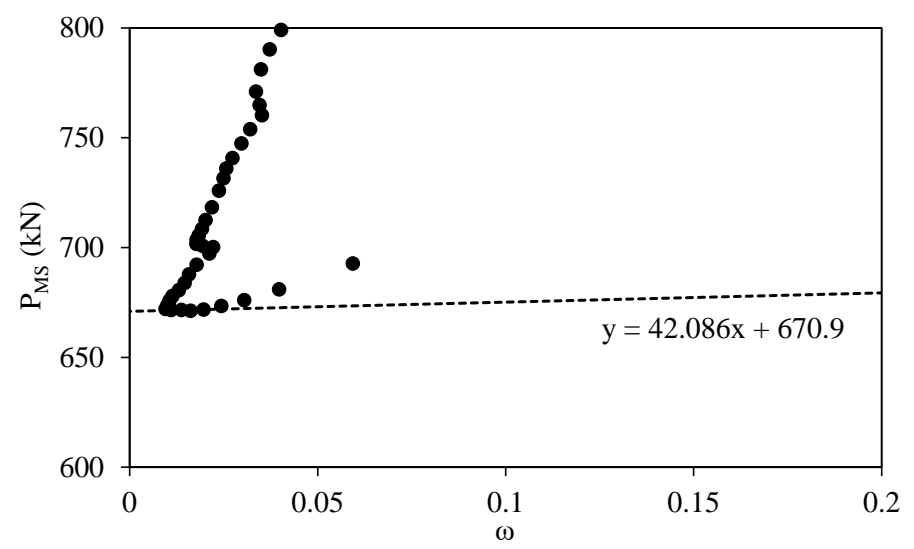

Fig. 9. Convergence Indicator Plot for a plate under a partial bearing load of $400 \mathrm{~mm}$.

Following the described approach, the CIP provided a plastic collapse load $P_{\mathrm{CIP}}$ of $670.9 \mathrm{kN}$. The predicted values of $P_{\mathrm{CIP}}$ and $P_{\mathrm{MS}}$ are almost identical, indicating that the both methods converge to the same collapse loads for the studied plate. Owing to its ability to provide a clear plateau for the identification of the plastic behaviour and its relative simplicity, the MS plot is recommended to obtain the plastic collapse loads from the MNA of steel members, and is used in the following sections to determine the plastic collapse loads of steel plates and cold-formed sections under concentrated loading.

\section{VERIFICATION OF PROPOSED PROCEDURE AND EXAMPLES}

In this section, the plastic collapse loads obtained from classical theoretical solutions of plates under axial loading and bending are used to verify the accuracy of the predicted plastic collapse loads obtained through the MS Plot. Following verification of the adopted procedure, the MS Plot is used to determine the plastic collapse loads of a series of plates under partial bearing loads and coldformed members under concentrated loading. 


\subsection{Verification of the adopted approach based on the MS Plot against theoretical solutions}

For the concentrically loaded plate shown in Fig. 10 (a), the theoretical plastic collapse load is equal to the product of the cross-sectional area and the yield stress. Adopting the same geometrical and material properties of the plate used in the Section 3.1, the theoretical plastic collapse load $P_{\mathrm{t}}$ can be determined as $1440 \mathrm{kN}$. The MS Plot obtained through MNA of the same plate is illustrated in Fig. 10 (b), along with the plastic collapse load obtained from the MS plot. As can be seen from Fig. 10 (b), the MS plot provides a collapse load equal to the theoretical value $P_{\mathrm{t}}$, which verifies the accuracy of the recommended procedure.

For the same plate subjected to an eccentric compressive loading with an eccentricity $r_{\mathrm{i}}$ of $20 \mathrm{~mm}$, as shown in Fig. 11 (a), the theoretical plastic collapse load can be determined considering the equilibrium of the plastic axial and bending stress diagrams. Assuming that the maximum longitudinal stress at yielding is limited to $2 / \sqrt{3} f_{y}$ due to the restrained anticlastic effect for the deformed plate in bending [10], the theoretical plastic collapse load of the plate can be determined by Eq. (1), where for $f_{y}=400 \mathrm{MPa}, \mathrm{L}=900 \mathrm{~mm}, \mathrm{t}=4 \mathrm{~mm}$ and $r_{i}=20 \mathrm{~mm}, \mathrm{P}_{t}=82.9 \mathrm{kN}$. The MS Plot obtained through MNA of the plate is illustrated in Fig. 11 (b), showing that the recommended MS procedure is able to provide a plastic collapse load prediction $(81.3 \mathrm{kN})$ that is almost the same as that determined through the theoretical expression.

$P_{t}=f_{y} L\left(\frac{-4 \sqrt{3} r_{i}+2 \sqrt{12 r_{i}^{2}+3 t^{2}}}{3}\right)$

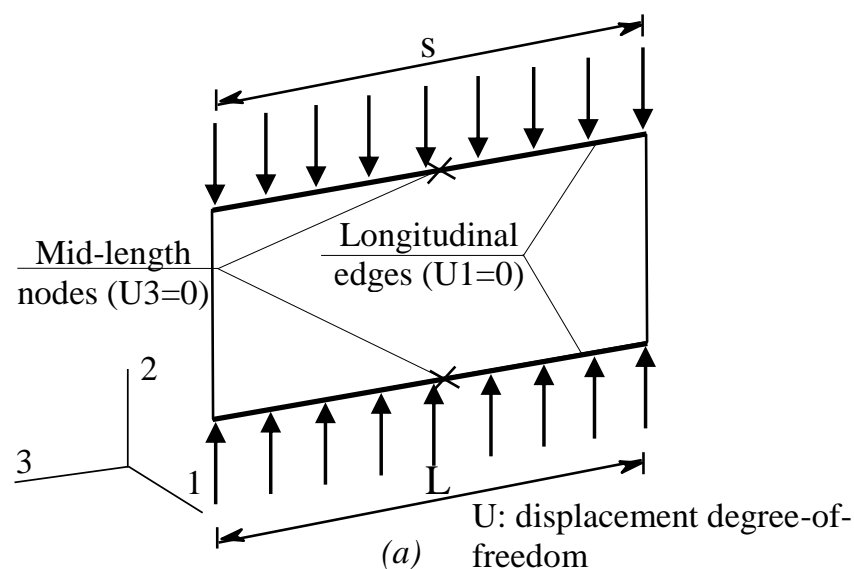

(a) freedom

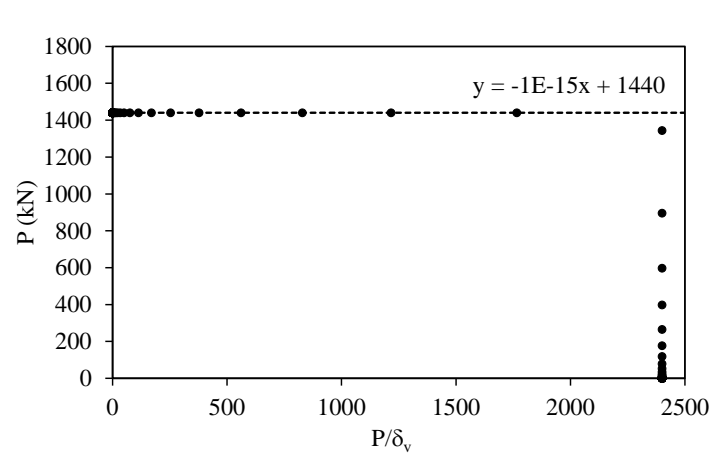

(b)

Fig. 10. (a) Boundary conditions of a uniformly and concentrically loaded plate and $(b)$ the MS Plot for this plate.
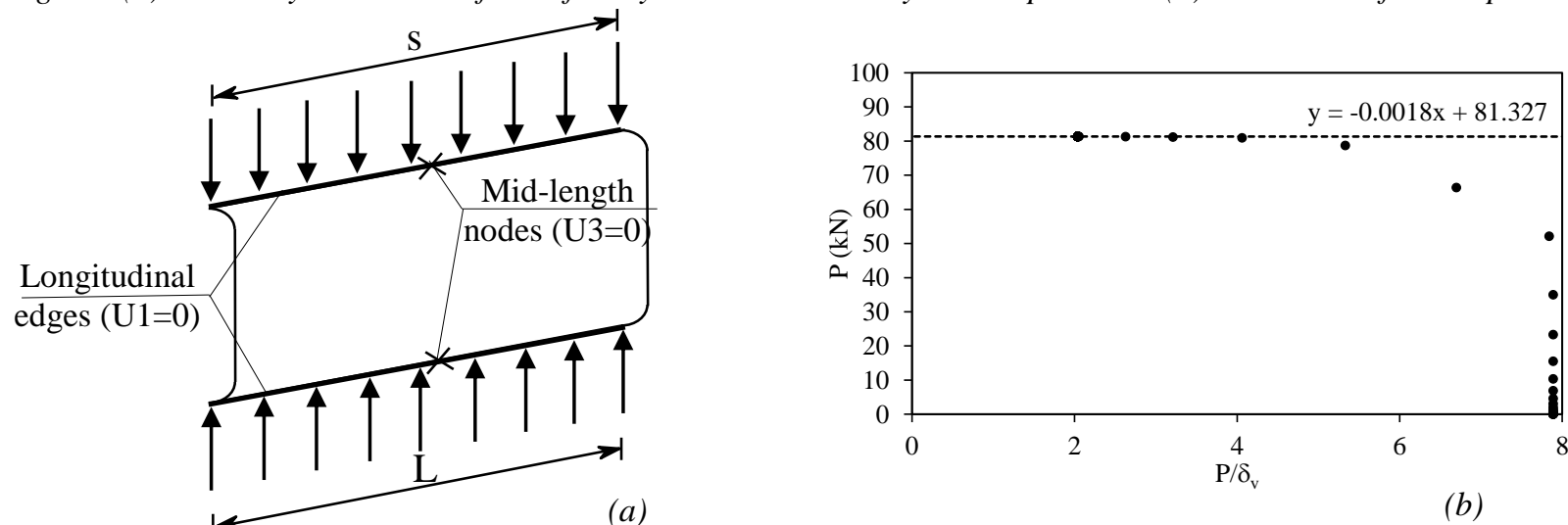

(a)

(b)

Fig. 11. (a) Boundary conditions of a uniformly and eccentrically loaded plate and (b) the MS Plot for this plate.

\subsection{Application of the MS plot to plates under partial bearing load and cold-formed sections}

In this section, the MS Plot is utilised to determine the plastic collapse loads of a series of steel plates subjected to partial bearing loads (i.e. plates with loaded lengths $s_{\mathrm{s}}$ less than the total edge lengths $L$ ) and those of cold-formed steel beams under concentrated transverse loads. 


\subsubsection{Plates subjected to different loaded lengths}

Fig. 12 shows the MS Plots of the rectangular plate studied in the previous subsection, considering different loaded lengths $s_{\mathrm{s}}$; the corresponding plastic collapse loads $P_{\mathrm{MS}}$ are also given. As can be seen from the figure and the table, particularly for the shorter loaded lengths, while the $\mathrm{P}_{\mathrm{MS}}$ values correspond to the extensive spread of plasticity (i.e. are representative of plastic collapse loads), they are much lower than the peak values from $P_{M N A}$, which are often obtained at excessively high deformations. Hence, simply taking the peak load from an MNA will yield unrealistic results.

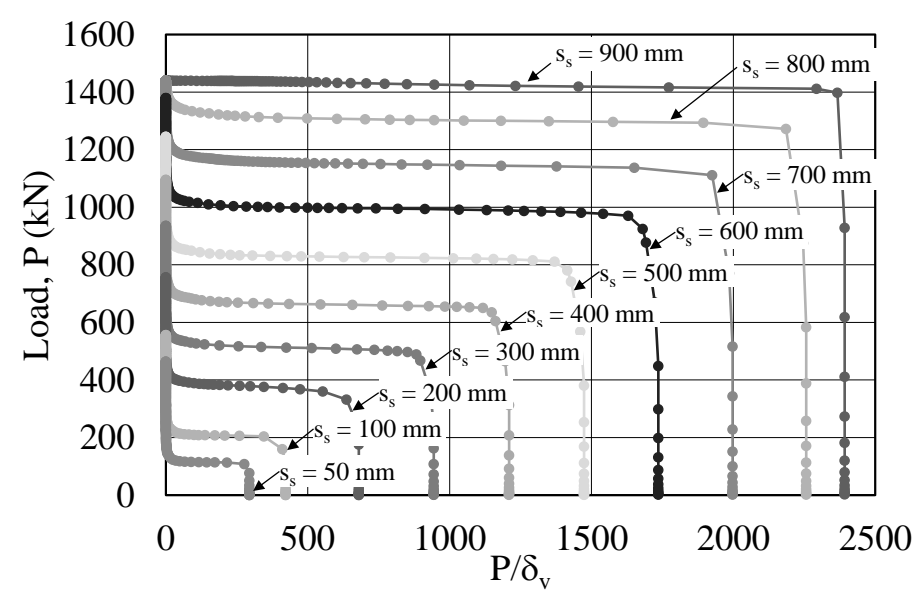

\begin{tabular}{ccc}
\hline $\mathrm{s}_{\mathrm{s}}(\mathrm{mm})$ & $\begin{array}{c}\text { Peak load from } \\
\text { MNA, } \\
\mathrm{P}_{\text {MNA }}(\mathrm{kN})\end{array}$ & $\begin{array}{c}\text { Predicted MS plastic } \\
\text { collapse load, } \\
\mathrm{P}_{\text {MS }}(\mathrm{kN})\end{array}$ \\
\hline 880 & 1440 & 1432 \\
\hline 800 & 1440 & 1310 \\
\hline 700 & 1440 & 1159 \\
\hline 600 & 1387 & 1003 \\
\hline 500 & 1245 & 835 \\
\hline 400 & 1100 & 671 \\
\hline 300 & 935 & 522 \\
\hline 200 & 756 & 390 \\
\hline 100 & 556 & 210 \\
\hline 50 & 464 & 116 \\
\hline
\end{tabular}

Fig. 12. Modified Southwell Plot applied to plates under partial bearing loads.

\subsubsection{Cold-formed elements under transversal concentrated loads}

In Fig. 13 (b), the MS plot for the cold-formed member under concentrated transverse loading analysed in the Section 3.2 is illustrated. Note that owing to convergence problems, the MNA of this member initially prematurely stopped prior to the development of a full plastic mechanism, precluding the determination of the plastic collapse load. As can be seen from Fig. 14, the MS Plot of the cold-formed member follows a trend similar to that observed in the previous cases with a quasi-vertical path where the load increases with small changes in the displacement and a plateau where plasticity develops with a significant increase in displacements. In this case, there exist two plateaus around the load values where two plastic zones develop; see Fig. 14.

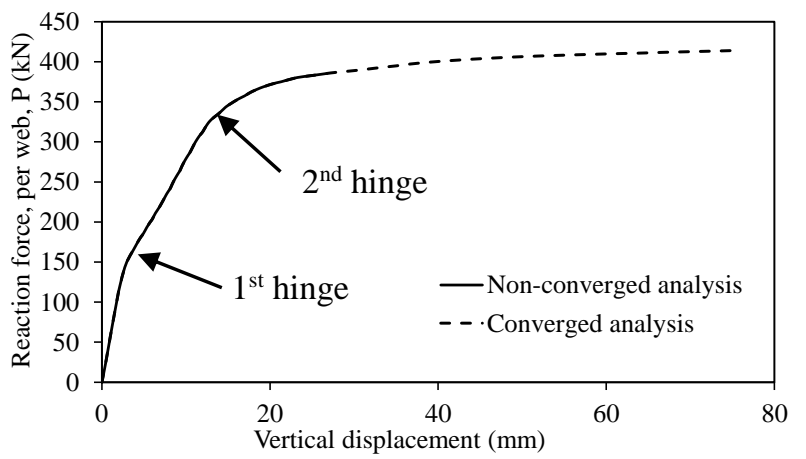

(a)

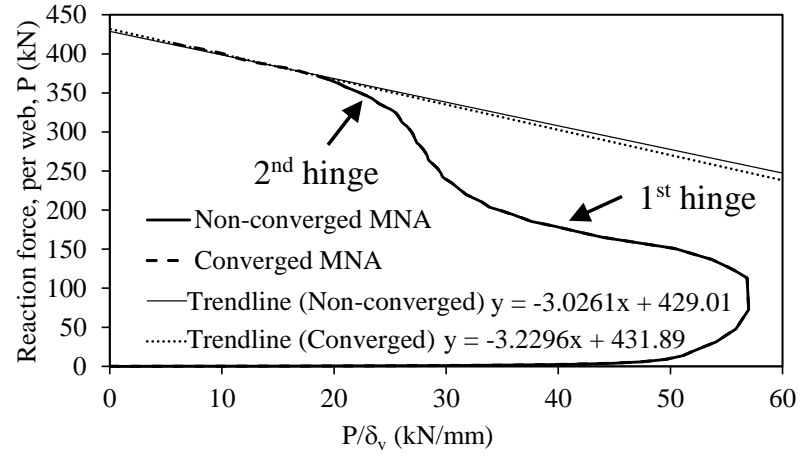

(b)

Fig. 13. Load-displacement and MS plot of a cold-formed member under partial bearing load (IOF250N90 [5]).

Following the procedure described in the previous section, the predicted plastic collapse load of the member was determined through regression lines fitted to three consecutive data points within the second plateau of the MS Plot, and found to be $P_{M S}=429 \mathrm{kN}$. To assess the accuracy of this value, the MNA of the member was also re-run, but with a reduced increment size, overcoming the convergence problem and obtaining a load displacement curve becoming flat at a load value very close to the predicted collapse load of $429 \mathrm{kN}$, which verifies the accuracy of the adopted approach. Note that in many cases, convergence problems persist, and hence use of an extrapolation techique such as that recommended in this section is required. 

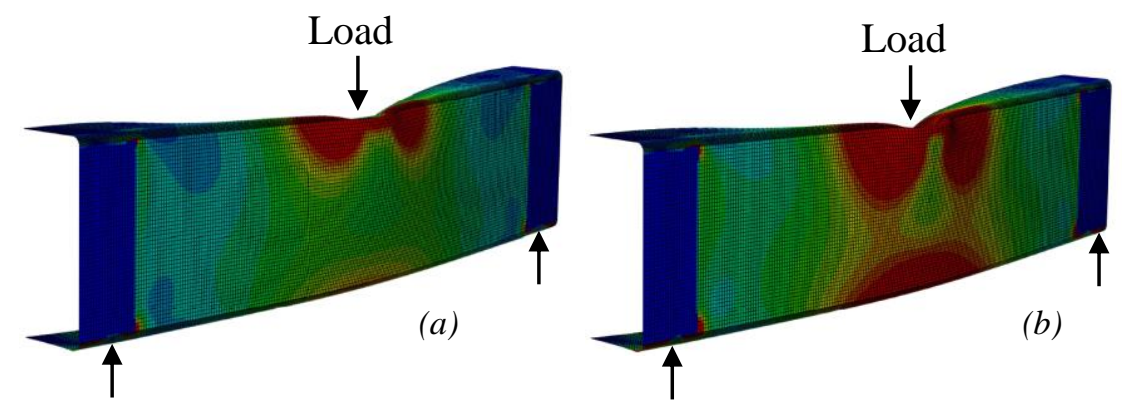

Fig. 14. MNA results for IOF250N90 - von Mises stress distribution for (a) $1^{\text {st }}$ yielded zone (b) $2^{\text {nd }}$ yielded zone.

\section{CONCLUSIONS}

Current structural steel design codes typically require the determination of the plastic collapse load and elastic critical load of steel elements, which are used to determine the slenderness and member resistance. When numerical analysis methods are employed, elastic critical loads are rather straightforward to calculate; however, in many cases, the determination of plastic collapse loads can be challenging. In this study, different extrapolation techniques that can be used to determine plastic collapse loads of steel elements from Materially Nonlinear Analyses (MNA) are investigated. Finite element models of steel plates and cold-formed members were created and validated against an experimental study from the literature. Two primary problems were encountered in the determination of the plastic collapse loads from MNA, which are (i) the development of collapse mechanisms only after unrealistic deformations and (ii) the premature termination of the MNA because of the failure to satisfy the necessary convergence criteria. Three different extrapolation techniques were investigated to overcome these problems: (i) the Southwell Plot, (ii) the Modified Southwell Plot (MS) Plot and (iii) the Convergence Indicator Plot (CIP). It was found that the plastic collapse loads of steel plates and cold-formed beams can be accurately predicted through the MS Plot with a relatively small amount of calculation effort; the predictions were verified against theoretical formulae, indicating that the recommended extrapolation techniques provide a practical and robust way to determine plastic collapse loads from MNA.

\section{ACKNOWLEDGMENTS}

The financial support of the Brazilian National Council of Technological and Scientific development $(\mathrm{CNPq})$ is gratefully acknowledged.

\section{REFERENCES}

[1] Duarte, A.P.C. and N. Silvestre, "A new slenderness-based approach for the web crippling design of plain channel steel beams". International Journal of Steel Structures, No 13(3): pp. 421-434, 2013.

[2] Bock, M. and E. Real, "Strength curves for web crippling design of cold-formed stainless steel hat sections". Thin-Walled Structures, No 85: pp. 93-105, 2014.

[3] SIMULIA. "ABAQUS Reference Manual 6.14". Dassault Systèmes [Software] 2014.

[4] Natário, P., N. Silvestre, and D. Camotim, "Computational modelling of flange crushing in cold-formed steel sections". Thin-Walled Structures, No 84: pp. 393-405, 2014.

[5] Young, B. and G.J. Hancock, "Design of cold-formed channels subjected to web crippling". Journal of Structural Engineering (ASCE), No 127(10): pp. 1137-1144, 2001.

[6] Doerich, C. and J.M. Rotter, "Accurate determination of plastic collapse loads from finite element analyses". Journal of Pressure Vessel Technology, No 133, 2011.

[7] Southwell, R.V., "On the analysis of experimental observations in problems of elastic stability". Proceedings of the Royal Society of London, Series A, No 135: pp. 601-616, 1932.

[8] Horne, M.R. and W. Merchant, "The stability of frames". London: Pergamon Press, 1965.

[9] Holst, J.M.F.G., C. Doerich, and J.M. Rotter. "Accurate determination of the plastic collapse loads of shells when using finite element analyses". in ICASS'05, Shanghai, China, 2005.

[10] Hiriyur, B.K.J. and B.W. Schafer, "Yield-line analysis of cold-formed steel members". Steel Structures, No 5: pp. 43-54, 2005. 